\title{
Intra-abdominal injury identified by CTA in stable blunt polytrauma patients
}

\author{
MC Mayet, ${ }^{1,2}$ TC Hardcastle, ${ }^{2,3}$ DJJ Muckart ${ }^{2,3}$ \\ 1 Department of Cardiothoracic Surgery, University of the Witwatersrand \\ 2 Inkosi Albert Luthuli Central Hospital, 800 Vusi Mzimela Rd, Mayville, 4058 \\ 3 Nelson R Mandela School of Medicine, University of KwaZulu-Natal
}

Corresponding author: Timothy Hardcastle (hardcastle@ukzn.ac.za)

\begin{abstract}
Background: Patients with multiple injuries are a challenge to evaluate and to exclude abdominal injury, especially those who are intubated and sedated. Ultrasound is a screening tool and peritoneal lavage is unreliable. The aim of the study was to determine the incidence of intra-abdominal injury and describe the subsequent management after CT "panscan" in patients sustaining blunt trauma with injuries both above the thoracic and below the pelvic diaphragm.

Methods: In a retrospective analysis anonymised patient data were extracted from a prospective ethics approved database of patients admitted to the level I Trauma Unit at Inkosi Albert Luthuli Central Hospital for the period from April 2007 to March 2011. Blunt polytrauma patients, aged 2 years and older with injuries above the diaphragm and below the pelvic floor were included, provided they were investigated by a full-body trauma Computed Tomography contrast study. Descriptive statistics were employed for all variables of interest, with counts/frequencies and associated percentages being reported.

Results: Of 284 patients with injuries above the thoracic and below the pelvic diaphragm, $87(30.6 \%)$ had intra-abdominal injury and $197(69.4 \%)$ had no intra-abdominal injuries. Of those 87 patients, $54(62.1 \%)$ were treated non-operatively and $33(37.9 \%)$ were treated surgically with regard to their abdominal injuries. Twenty $(22.9 \%)$ patients died, 4 due to intraabdominal injuries and 16 due to of extra-abdominal injuries. Nine (45\%) of the twenty patients who died were treated operatively for intra-abdominal injuries and the remaining $11(55 \%)$ were treated non-operatively.

Conclusion: Around thirty percent of patients with injuries above the thoracic and below the pelvic diaphragm had concomitant intra-abdominal injuries. Of those with abdominal injury, just over half required laparotomy. For haemodynamically stable patients CT scanning identified those who require surgical intervention and those who may be managed non-operatively, therefore liberal CT-scanning is advisable for this patient group.
\end{abstract}

Keywords: blunt trauma, CT, diaphragm, pelvic diaphragm, abdominal injury

S Afr J Surg 2019;57(1)

http://dx.doi.org/10.17159/2078-5151/2019/v57n1a2715

\section{Introduction}

The evaluation of patients who have sustained blunt abdominal trauma may pose a significant diagnostic challenge to even the most seasoned trauma surgeon. Blunt trauma produces a spectrum of injury from minor, singlesystem injury to devastating, multi-system trauma. While a carefully performed physical examination remains essential in determining the need for exploratory laparotomy, there is little level I evidence to support this tenet. ${ }^{1}$ In fact, several studies have highlighted the inaccuracies of the physical examination in blunt abdominal trauma. ${ }^{1}$ The inaccuracy of abdominal examination in eliciting signs of peritoneal irritation in injured patients is considerable: a false positive rate of $40-60 \%$ is usual, while the false negative rate is $30-50 \% .^{2}$ This is especially true in those with an altered level of consciousness or distracting injuries out of the abdomen. These missed injuries are associated with a considerable increase in mortality. ${ }^{2,3,4}$

Prior to computerised scanning (CT), where diagnostic doubt remained following clinical examination, diagnostic peritoneal lavage (DPL) was adopted to determine the presence of intra-abdominal injury. ${ }^{1}$ Although the high sensitivity and specificity results in the identification of virtually all intraperitoneal injuries, many minor grades of solid organ injury do not require surgical intervention. Furthermore, DPL fails to assess retroperitoneal injuries. Focussed abdominal sonar for trauma (FAST) will identify the presence of free fluid but not the specific injury and has the same disadvantage as DPL for retroperitoneal trauma. ${ }^{1} \mathrm{CT}$, designed by the combined efforts of Hounsfield and Cormack, revolutionised diagnostic interventions in innumerable 
specialities. Although less sensitive in detecting hollow visceral intra-abdominal injury, it assesses both the intra- and extraperitoneal compartments, and allows solid organ injuries to be graded thus facilitating management. ${ }^{1}$ Furthermore, in those sustaining multisystem trauma, CT scanning is essential in evaluating traumatic brain and thoracic injury.

Concern has been expressed regarding the radiation exposure during CT angiography and the induction of later malignancy. Recent publications have suggested that in victims of multisystem trauma selective rather than wholebody CT scanning may be appropriate, thus reducing this theoretical risk. ${ }^{5}$ The potential disadvantage is the threat of a missed injury and diagnostic delays. If selective CT scanning is to be employed, then the incidence of intra-abdominal injury needs to be ascertained before such conservatism may be introduced safely, and in particular, those requiring surgical intervention. With that in mind, a review of all patients who had sustained blunt polytrauma and undergone CT angiography was undertaken.

\section{Methods}

The Trauma Unit at Inkosi Albert Luthuli Central Hospital (IALCH), Durban, a major regional referral hospital, is an accredited level 1 trauma centre, including an integrated Trauma ICU (TICU). The unit has 8 ICU beds and 2 high care beds, and admits both adult and paediatric patients above the age of 2 years. The trauma unit accepts patients directly from the scene of the incident as well as from drainage hospitals after patients have been stabilised. Polytrauma patients who are haemodynamically stable are investigated by whole body CT angiography as a routine, either at the base hospital or at IALCH.

After obtaining approval from the University of the Witwatersrand Human Research Ethics Committee (Clearance certificate no. M130712), data were extracted from the UKZN Ethics Committee (BCA207/09) approved prospective database, for patients admitted to the TICU at IALCH for the period April 2007-March 2011. The rights and confidentiality of the patients were protected by abstracting only anonymised data from the Microsoft Excel Spreadsheet (Microsoft Corp, Redmond WA) Trauma unit registry. Blunt polytrauma patients, over the age of 2 years, with injuries both above the diaphragm and below the pelvic floor were included, provided they were investigated by a full-body trauma CT with angiography. Those patients who underwent emergency laparotomy on clinical grounds, or FAST examination only, were excluded. Descriptive statistics were employed for all variables of interest, with counts/frequencies and associated percentages being reported, using the GraphPad ${ }^{\circledR}$ (Graphpad Inc, CA, USA).

\section{Results}

A total of 284 patients were identified in the Trauma Registry with injuries both above the thoracic diaphragm and below the pelvic diaphragm of whom 197 (69.3\%) did not have any intra-abdominal injuries and 87 (30.6\%) had CT evidence of intra-abdominal injury. Mechanism of injury and demographics are shown in Table 1. It is interesting to note that certain mechanisms of injury are associated with a high risk of intra-abdominal pathology, including pedestrianvehicle collisions (62\%) and vehicular occupants $(28.7 \%)$.

Figure 1 illustrates the abdominal injuries and the management undertaken at the unit. There were 112 injuries of which solid organ injuries accounted for 80 (71.4\%). The commonest solid organ to be injured was the liver in $34(39.1 \%)$ patients of which $30(88.2 \%)$ were successfully managed non-operatively. Thirty $(34.5 \%)$ patients suffered splenic trauma of which $5(16.6 \%)$ required splenectomy. There were 15 renal injuries with $4(26.6 \%)$ patients necessitating nephrectomy. Three (3.4\%) patients suffered diaphragmatic injuries, which were all managed operatively and $1(1.1 \%)$ patient sustained a pancreatic injury that was

Table 1. Mechanism of injury and patient demographics

\begin{tabular}{lcc}
\hline Injury Mechanism & No Abdominal Injury & \multicolumn{2}{c}{$\begin{array}{c}\text { Abdominal Injury } \\
\text { \& Demographics }\end{array}$} & $\mathbf{( N = 1 9 7 )}$ & $51(58.6 \%)$ \\
\hline Male & $131(66.4 \%)$ & $36(41.3 \%)$ \\
Female & $66(33.5 \%)$ & $38(2-68)$ years \\
Age (Median and Range) & $41(5-80)$ years & $124(102-134) \mathrm{mmHg}$ \\
SBP (Median and IQR) & $128(107-143)$ mmHg & $2.5(1.0-2.9)$ \\
Lactate (Mean / SD) & $2.3(0.6-3.2)$ & $20(22.9 \%)$ \\
Deaths & $40(20.3 \%)$ & $54(62 \%)$ \\
Pedestrian Vehicle Collision & $88(44.6 \%)$ & $25(28.7 \%)$ \\
Vehicle Occupant & $92(46.7 \%)$ & $2(2.2 \%)$ \\
Motor Cycle & $7(3.5 \%)$ & $1(1.1 \%)$ \\
Pedal Cycle & $1(0.5 \%)$ & $5(5.7 \%)$ \\
Non-vehicular & $9(12.2 \%)$ & $17(19.5 \%)$ \\
Referred from scene & $75(38 \%)$ & $65(74.7 \%)$
\end{tabular}


managed conservatively. Of hollow visceral injuries the bladder was the commonest, occurring in $9(10.3 \%)$ patients with intra-abdominal injury, followed by small intestinal injuries in $7(8 \%)$ patients, large bowel injuries in $3(3.4 \%)$ patients and stomach injury in $1(1.1 \%)$. Intra-abdominal vascular injuries included severe pelvic fractures with $>20 \%$ blood loss in $3(3.4 \%)$ patients, mesenteric vascular injury in $3(3.4 \%)$ patients, IVC injury in $2(2.2 \%)$ patients, and aortic injury in $1(1.1 \%)$ patient.

Of the 87 patients with concomitant intra-abdominal injuries, $67(77 \%)$ survived to discharge and 20 (22.9\%) died. Four patients died as a result of their intra-abdominal injuries and 16 died as a result of injuries to other body regions. Cause of death in the 4 patients who died as a direct result of intra-abdominal injuries were: massive blood loss as a result of open book pelvic fractures in 2 cases, however both had arrived post-damage control haemostatic surgery (pelvic packing) at base-hospitals and were stable for scan as completion of secondary survey to identify all other injuries. One other patient had delayed presentation to IALCH as a transfer, with an iliac artery injury and limb ischaemia, and finally one patient with septic shock due to disembowelment that was managed using a Bogota bag and subsequently complicated with intra-abdominal sepsis. Of the 67 patients who survived to discharge, 43 were treated non-operatively and 24 were treated surgically.

\section{Discussion}

Following polytrauma, serial abdominal examinations are often not possible as patients may have an altered level of consciousness due to traumatic brain injury, anaesthesia for operative procedures on other injuries, or sedation once transferred to the intensive care unit. ${ }^{3}$ Further difficulties arise when the patient has an injury to their spinal cord or adjacent structures such as the lumbar spine or lower rib cage. All these factors have led to a more liberal use of diagnostic imaging for trauma patients with suspected abdominal injuries. Due to the recognised inadequacies of physical examination,

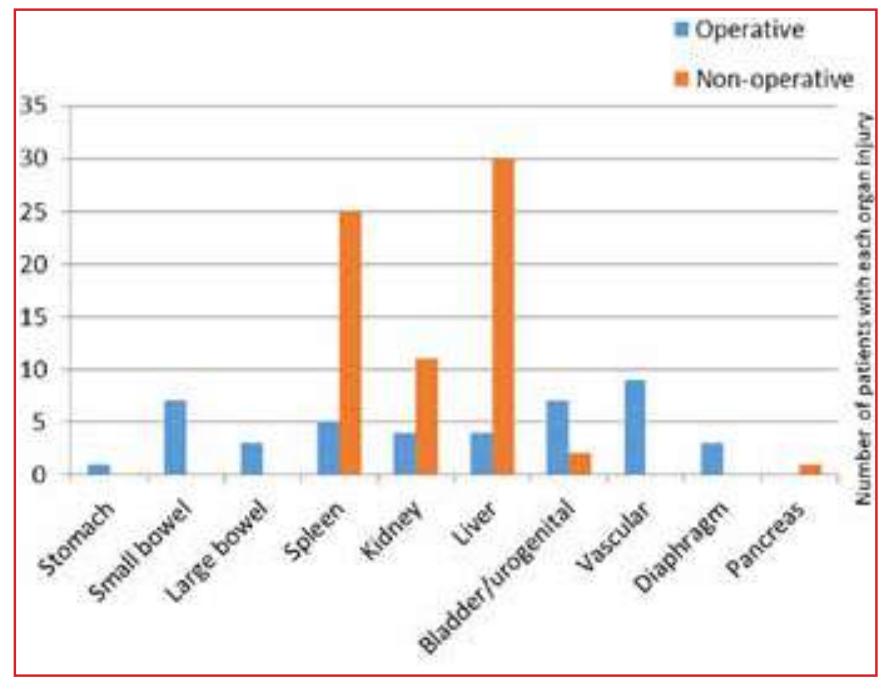

Figure 1. Organ injuries and management thereof trauma surgeons have come to rely on a number of diagnostic adjuncts.

Several factors influence the selection of diagnostic testing: (1) type of hospital - i.e., trauma center vs. "non-trauma" hospital; (2) access to a particular technology at the surgeon's institution; or (3) the surgeon's individual experience with a given diagnostic modality. ${ }^{1}$ As facilities evolve, technologies mature and surgeons gain new experience, it is important that any diagnostic strategy constructed be dynamic and adaptable to that particular institution. No injury can be considered in isolation: the management and investigation of abdominal trauma should be conducted along systematic lines such as those described in the Advanced Trauma Life Support (ATLS) courses. $^{2}$ In the majority of cases, time is available for the assessment of the abdomen, but it should be emphasised that all too often abdominal injuries are missed, or only diagnosed, after undue delay. ${ }^{2} \mathrm{~A}$ high degree of suspicion must be maintained when dealing with any injured patient with a history that could even remotely be associated with abdominal injury, even in the absence of overt signs.

The primary purpose of this study was to determine the incidence of intra-abdominal injury in the polytrauma patient with clinically, or radiographically obvious injuries, both above the thoracic diaphragm and below the pelvic diaphragm. Secondary objectives included a description of the mechanisms of injury, a description of the abdominal injuries sustained, and determining management of injuries (operative versus non-operative). The findings could provide guidelines for determining how extensively these patients should be investigated with regard to their possible abdominal injuries. The vast majority of patients in this study were routinely investigated via $\mathrm{CT}$, which required a haemodynamically stable patient (defined as blood pressure $>100 \mathrm{mmHg}$ systolic, pulse rate $<110 / \mathrm{min}$ and lactate $<4 \mathrm{mmol} / \mathrm{l}$, without clinically active bleeding), and almost $31 \%$ were found to have intra-abdominal injury, particularly those injured as pedestrians stuck by a vehicle (62\%). In addition, the patients were transported out of the trauma resuscitation area to the Radiology department, accompanied by the trauma team. These factors are viewed as limitations to the utility of CT for trauma patients. ${ }^{2}$ Specialised technicians and the availability of a radiologist for interpretation are also required.

CT scanners are now available in most trauma centers and, with the advent of helical scanners, scan time has been significantly reduced. ${ }^{1}$ As a result, CT has become an accepted part of the trauma surgeon's armamentarium. CT has replaced DPL as the imaging method of choice in many trauma centers worldwide. Its major advantage is that it is not only capable of revealing the presence of intra-abdominal or intra-thoracic haemorrhage but can mostly identify and grade injury severity of the organ involved. ${ }^{4}$ The accuracy of CT in haemodynamically stable blunt trauma patients has been well established. Sensitivity between $92 \%$ and $97.6 \%$ and specificity as high as $98.7 \%$ have been reported in patients subjected to emergency $\mathrm{CT} .{ }^{1} \mathrm{CT}$ is notoriously inadequate for the diagnosis of mesenteric injuries and may also miss up to $15 \%$ of hollow visceral injuries. ${ }^{6}$ In patients at risk for 
mesenteric or hollow visceral injury, DPL is generally felt to be a more appropriate test. A negative CT scan in such a patient cannot reliably exclude intra-abdominal injuries. ${ }^{1,6} \mathrm{CT}$ has the unique ability to detect clinically unsuspected injuries. ${ }^{7}$ Additional advantages of CT scanning over other diagnostic modalities are the ability to evaluate the retroperitoneal structures and rapid scan time. ${ }^{8,9}$

Published work suggests a survival benefit for the trauma patient who underwent total-body CT scanning during the initial trauma assessment. ${ }^{5}$ Total-body imaging is theoretically so promising that several trauma centres around the world have incorporated the total-body CT scan into their daily practice, without level 1 evidence. ${ }^{5}$ In a recent randomised multicentre trial, no significant difference was found in in-hospital mortality in patients with severe trauma who underwent immediate total-body CT scanning compared to the standard work-up with conventional imaging and selective CT scanning. ${ }^{5}$ Although the radiation dose was increased in those undergoing total-body CT group compared to the selective group, the time to diagnosis of all injuries to the end of imaging in the trauma room was shorter with total-body CT scanning. ${ }^{5}$ The estimated risk of radiation-induced malignancy is in the region of $0.18 \%$ for a one-year-old undergoing CT scanning..$^{10}$ Firstly, that is substantially lower than fatalities from missed injuries and secondly, the vast majority of trauma patients are much older, and the dose to mass ratio is lower thereby reducing the radiation risk. ${ }^{11}$ Furthermore, $46 \%$ of patients in the selective group eventually underwent total body CT by sequential scanning. The authors used an intention to treat analysis where if the crossover rate is more than $10 \%$, a per protocol or as treated analysis is recommended. This was not undertaken making the validity of the results questionable. There was no cost difference between the groups. ${ }^{5}$

In their summary of the REACT-2 study, the authors suggest that "...large prospective series of haemodynamically unstable patient cohorts should be done to provide information on the transition point between those who are unstable but stable enough for a total-body CT scan and those who are too unstable to undertake a total-body CT scan". 5 They have provided no evidence to support this statement and we would urge extreme caution. It is well known to all trauma surgeons that unless there are valid reasons to suspect that hypotension is not due to ongoing haemorrhage, subjecting a haemodynamically unstable patient to imaging outside the resuscitation area is foolhardy and associated with an increased mortality. ${ }^{12}$

In recent years, focused abdominal sonography for trauma (FAST) has emerged as a useful diagnostic test in the evaluation of blunt abdominal trauma. The advantages of the FAST examination have been clearly established. FAST is non-invasive, may be easily performed and can be executed concurrently with resuscitation. In addition, the technology is portable and may be easily repeated if necessary. In most cases, FAST may be completed within 3-4 minutes. The test is especially useful for detecting intra-abdominal haemorrhage in the multiply injured or pregnant patient. A noted drawback to the FAST examination is the fact that a positive examination relies on the presence of free intraperitoneal fluid. In the hands of most operators, ultrasound will detect a minimum of $200 \mathrm{ml}$ of fluid. Injuries not associated with haemoperitoneum may not be detected by this modality. Thus, it is not a reliable method for excluding hollow visceral injury. In addition, the FAST examination cannot be used to reliably grade solid organ injuries.

FAST compares favourably with other traditionally utilised diagnostic tests. ${ }^{1}$ In the haemodynamically stable patient, FAST offers a reasonable alternative to DPL. DPL may also be used as a complementary examination in the haemodynamically stable patient in the presence of an equivocal or negative ultrasound with strong clinical suspicion of visceral injury. FAST has demonstrated utility in haemodynamically stable patients. In addition, ultrasound has been shown to be more cost-effective when compared to DPL or CT. The level of accuracy is independent of the practitioner performing the study, with surgeons, emergency medicine physicians, ultrasound technicians and radiologists having equivalent results. ${ }^{1}$

While serious intra-abdominal trauma commonly occurs with isolated injuries to the chest and pelvis, the incidence of abdominal injury may not be greatly increased in patients with combined injuries to the chest and the pelvis. However, one previous study lends support to the argument that those patients with severe chest and pelvis injuries should have their abdominal cavity imaged to exclude concomitant serious abdominal injury..$^{13}$ Patients whose clinical condition dictates that they receive immediate emergency surgery should not be delayed by unnecessary imaging.

Standard imaging and CT scanning must not be considered competitive but complementary. Furthermore, it must be understood that a negative CT scan should not be viewed as a failure of clinical acumen or protocol, but provides as much useful information as a positive diagnosis. ${ }^{14}$ In addition, the model used to estimate the risk of radiation-induced malignancy has recently been considered invalid and should not be used as a contraindication to CT scanning. ${ }^{15}$ The ability to grade organ injury is the cornerstone of selective non-operative management and reduces the non-therapeutic laparotomy rate with its considerable associated morbidity.

\section{Conclusions and recommendations}

Given the findings in the current study of a $30 \%$ incidence of intra-abdominal injuries in those patients with trauma both above the thoracic and below the pelvic diaphragms and that CT allowed almost two-thirds to be managed non-operatively, we recommend CT scanning in all such patients. Additionally, given the high positive CT-scan rate for pedestrian collision victims $(62 \%)$, CT should become a routine practice in such patients if they are haemodynamically stable. Future prospective studies of both haemodynamically stable and unstable patients may give clearer definition of the timing and role of CT-scan for these patients.

Study limitations include the fact that this was a singlecentre study, albeit using an electronic patient record system and prospectively collected data in the registry. The potential 
of recording bias due to the timing of note-making by the clinicians on the electronic record system is a possibility, as is the potential for missing data, despite the use of notetemplates.

This work was not funded and there are no conflicts of interest to declare

\section{REFERENCES}

1. Hoff WS, Holevar M, Nagy KK, et al. Practice management guidelines for the evaluation of blunt abdominal trauma; EAST Practice Management Guidelines Work Group. J Trauma. Sep 2002;53(3):602-15.

2. Anderson I, Irving M. The investigation of abdominal trauma. Arch Emerg Med. 1990;7:1-8.

3. Hardcastle TC. Missed injury - decreasing morbidity and mortality: A literature review. S Afr J Surg. 2011;49:199-201.

4. Steinwall D, Befrits F, Naidoo SR, et al. T. Deaths at a Level 1 Trauma Unit: A clinical finding and post-mortem correlation study. Injury. 2012;43:91-5.

5. Sierink JC, Treskes J, Edwards MJR, et al. Immediate totalbody CT scanning versus conventional imaging and selective CT scanning in patients with severe trauma (REACT-2): a randomised controlled trial. Lancet. 2016;388:673-83.

6. Banz VU, Butt MU. Free abdominal fluid without obvious solid organ injury upon CT imaging: an actual problem or simply over-diagnosing? J Trauma Manag Outcomes. 2009 [accessed on 14 Apr 2011];3:10. doi: 10.1186/1752-2897-3-10

7. Smith CB, Barrett TW, Berger CL, et al. Prediction of blunt traumatic injury in high-acuity patients: bedside examination vs computed tomography. Am J Emerg Med. 2011;29:1-10.

8. Zwingmann J, Schmal H, Mehlhorn A, et al. Injury patterns in polytraumatized children and consequences for the emergency room management. Acta Chir Orthop Traumatol Cech. 2010;77:365-70.

9. Milla DJ, Brasel K. Current use of CT in the evaluation and management of injured patients. Surg Clin N Am. 2011;91:23348.

10. Brenner D, Elliston C, Hall E, Berdon W. Estimated risks of radiation-induced fatal cancer from pediatric CT. Am J Roentgenol. 2001;176:289-96.

11. Muckart DJJ, Thomson SR. Undetected injuries: a preventable cause of increased morbidity and mortality. Am J Surg. 1991;162:457-60.

12. Neal MD, Peitzman AB, Forsythe RM, et al. Over reliance on computed tomography imaging in patients with severe abdominal injury: is the delay worth the risk? J Trauma. 2011;70:278-84.

13. Cooper JG, Smith R, Cooper AJ. The incidence of abdominal injury in patients with thoracic and/or pelvic trauma. Injury Extra. 2005;36:259-63.

14. Oosthuizen GV, Bruce JL, Bekker W, et al. Pan computed tomography for blunt polytrauma: Are we doing too many? S Afr Med J. 2016;106:801-3.

15. Siegel JA, Pennington CW, Sacks B, Welsh JS. The Birth of the Illegitimate Linear No-Threshold Model: An Invalid Paradigm for Estimating Risk Following Low-dose Radiation Exposure. Am J Clin Oncol. 2018;41:173-7. 